\title{
BMJ Open Evolution of epilepsy prevalence and incidence in a Tanzanian area endemic for onchocerciasis and the potential impact of community-directed treatment with ivermectin: a cross-sectional study and comparison over 28 years
}

Helena Greter, ${ }^{1}$ Bruno Mmbando, ${ }^{2}$ Williams Makunde, ${ }^{2}$ Mohamed Mnacho, ${ }^{3}$ William Matuja, ${ }^{3}$ Advocatus Kakorozya, ${ }^{4}$ Patrick Suykerbuyk, ${ }^{1}$ Robert Colebunders ${ }^{1}$

To cite: Greter H, Mmbando B, Makunde W, et al. Evolution of epilepsy prevalence and incidence in a Tanzanian area endemic for onchocerciasis and the potential impact of community-directed treatment with ivermectin: a crosssectional study and comparison over 28 years. BMJ Open 2018;8:e017188. doi:10.1136/ bmjopen-2017-017188

- Prepublication history and additional material for this paper are available online. To view these files, please visit the journal online (http://dx.doi. org/10.1136/bmjopen-2017017188).

Received 7 April 2017 Revised 11 February 2018 Accepted 22 February 2018

Check for updates

${ }^{1}$ Global Health Institute, University of Antwerp, Wilrijk, Belgium

${ }^{2}$ National Institute of Medical Research, Tanga Research Centre, Tanga, Tanzania ${ }^{3}$ Department of Neurology, Muhimbili University of Health Sciences, Dar es Salaam, Tanzania

${ }^{4}$ Enhance Tanzania Foundation, Dar es Salaam, Tanzania

Correspondence to Prof Robert Colebunders; robert.colebunders@ uantwerpen.be

\section{ABSTRACT}

Introduction Worldwide, there are an estimated 50 million people affected by epilepsy. Its aetiology is manifold, and parasitic infections play an important role, specifically onchocerciasis. In onchocerciasis endemic areas, a distinctive form of epilepsy has been described as nodding syndrome, affecting children and causing nodding seizures, mental retardation and debilitating physical development. Onchocerciasis control programmes using community-directed treatment with ivermectin (CDTI) are implemented in endemic countries. This study is designed to contribute to a better understanding of the linkage between the onset of epilepsy, onchocerciasis and CDTI. Comparing the epidemiological data on epilepsy and onchocerciasis from pre-CDTI and 20 years after its introduction will allow identifying a potential impact of ivermectin on the onset of epilepsy.

Methods and analysis The study will be conducted in the Mahenge highlands in Tanzania. Study site selection is based on an in-depth study on epilepsy in that area dating from 1989. CDTI was introduced in 1997. By a door-to-door approach, the population will be screened for epilepsy using a validated questionnaire. Suspected cases will be invited for a neurological examination for case verification. Onchocerciasis prevalence will be assessed by a rapid epidemiological assessment. As an indicator for ongoing transmission, children younger than 10 years of age will be tested for 0v16 antibodies. Ivermectin use will be assessed at household level. Epilepsy data will be analysed in comparison with the 1989 data to reveal pre-CDTI and post-CDTI prevalence and incidence.

Ethics and dissemination The protocol has received ethical approval from the ethics committees of the University of Antwerp, Belgium, and of the National Institut of Medical Research, Dar es Salaam, Tanzania. The findings will be published in peer-reviewed journals, and presented to the health authorities in Tanzania, at national, regional and village level.

\section{Strengths and limitations of this study}

- This study will allow comparison of large-scale population-based data on epilepsy epidemiology in Mahenge over a period of 28 years.

- The strength of the study is that it is a first study to follow-up epilepsy epidemiology in the Mahenge area since the introduction of community-directed treatment with ivermectin (CDTI).

- The comparaison of the data obtained in 1989 and 2017 will be challenged by the slightly different study methodologies used.

- The study design is limited in accounting for potential confounding factors other than CDTI that may influence epilepsy incidence and prevalence.

- Focusing on villages with high epilepsy burden in the past may lead to an overestimation of the potential impact of CDTI.

\section{INTRODUCTION}

Epilepsy in onchocerciasis endemic regions in Africa

Epilepsy is a chronic disease estimated to affect 50 million people worldwide according to WHO. ${ }^{1}$ In general, higher prevalence and incidence are reported among those populations living in low-income and middle-income countries (LMICs) when compared with industrialised countries. ${ }^{23}$ In fact, more than $85 \%$ of the global burden of epilepsy occurs in people living in LMICs. ${ }^{45}$ The aetiology of epilepsy is very diverse and not yet fully understood. Besides birth trauma and head injury involving the brain, infectious diseases can trigger epilepsy as well. Several parasitic infections are associated with epilepsy such as cerebral malaria, neurocysticercosis, echinococcosis and onchocerciasis. ${ }^{6-8}$ Many of 
these epilepsy cases could be prevented through timely antiparasitic treatment. Epilepsy manifests in a variety of seizure types and intensity. ${ }^{9}$ In 1960 in Tanzania, Aall-Jillek was the first to describe an unusual form of epileptic seizures characterised by nodding movements of the head. ${ }^{10}{ }^{11}$ Later, this distinct form of epilepsy has been described also in Uganda and South Sudan and has been named as nodding syndrome (NS) ${ }^{12}{ }^{13} \mathrm{NS}$ is a debilitating epileptic disorder mainly affecting children at the ages of 3 and 18 years. ${ }^{14}$ The seizures are characterised by a brief loss of muscle tone in the neck, leading to repetitive head-nodding. ${ }^{15} \mathrm{NS}$ is often associated with cognitive decline and sometimes with stunted growth. ${ }^{16}$ So far, NS is solely described in onchocerciasis endemic areas. ${ }^{12}$ An NS epidemic has been observed in the past two decades in northern Uganda and in neighbouring South Sudan. ${ }^{16} 17$ The weight of the public health burden caused by epilepsy in onchocerciasis endemic regions can be illustrated by the situation in the village Mvolo (West Equatorial State in South Sudan) over $50 \%$ of the families had at least one child with epilepsy, resulting in one in six children of the village suffering from epilepsy. ${ }^{18}$

Health systems services in remote rural regions in Africa are rarely capable to provide continuous antiepileptic treatment to those patients in need. ${ }^{5}$ Furthermore, most healthcare workers lack training to diagnose and treat persons with epilepsy adequately. In addition to its clinical burden, epilepsy is perceived as a possession by evil spirits in many African societies and hence bears a stigma that puts the diseased individual and his family at risk for social isolation. ${ }^{19}$ Moreover, the family's economy is negatively impacted by the disease, since an epileptic family member needs specific care and supervision, detaining care takers from their subsistence duties. ${ }^{20}$

NS only occurs in onchocerciasis endemic areas. An epidemiological association between epilepsy and onchocerciasis was first reported from Western Uganda in the early 1990s. ${ }^{21} 22$ A case-control study performed in 1991-1992 in the Mbam valley in Cameroon demonstrated a significantly higher microfilarial load in persons with epilepsy than in controls. ${ }^{23}$ Study results from other onchocerciasis endemic African countries underline this association. ${ }^{824}$ To describe this epidemiological phenomenon, the term onchocerciasis-associated epilepsy (OAE) was proposed by Kaiser and colleagues. ${ }^{24}$ Case-control studies in northern Uganda and South Sudan focusing on patients with NS produced similar results, showing a higher prevalence of onchocerciasis in NS cases compared with non-epileptic controls. ${ }^{16}{ }^{17}$ It is, however, unclear how onchocerciasis might cause NS. Although the eye and the optical nerve are affected when onchocerciasis causes blindness, microfilariae and adult Onchocerca volvulus worms are not generally considered to be able to invade the central nervous system. Recent research hypothesises that an immunological cross-reaction of onchocerciasis-specific antibodies may provoke a neurotoxic reaction and trigger $\mathrm{NS}^{25}$
Onchocerciasis: a treatable neglected tropical disease facing obstacles on the road to elimination

Onchocerciasis is a parasitic disease caused by an infection with the worm $O$. volvulus whose filarial larvae are transmitted by blackflies (Simulidaespp.). In the final host (humans), the adult female worms encapsulate in the subcutaneous tissue forming visually detectable nodules. Each female worm releases up to one thousand microfilariae per day. These microfilariae provoke itching, dermatitis and-if left untreated-blindness, leading to the disease name river blindness. Blackflies get infected with microfilariae when biting infected humans in proximity to fast-flowing rivers, the breeding site of the blackflies. After passing through several larval stages, infected blackflies disseminate the parasite by biting other people. Onchocerciasis is treatable with ivermectin. ${ }^{26}$ The drug has a twofold mechanism of action: (1) it kills the microfilariae and (2) inhibits their release by the adult female worm for several months up to 2 years after a single dose treatment. ${ }^{27}$ Hence, ivermectin has a strong impact on reducing transmission. However, ivermectin is not lethal to adult worms, and infected persons have to be treated annually for up to 15 years. ${ }^{28}$ Onchocerciasis is a priority disease scheduled for elimination by 2025 by the WHO. Today, $99 \%$ of the globally 37 million people infested live in Africa. ${ }^{1}$ In 1995, the African Program for Onchocerciasis Control (APOC) was initiated for the implementation of the onchocerciasis control programme based on community-directed treatment with ivermectin (CDTI). APOC was coordinating these activities in endemic areas of 22 African countries. ${ }^{29}$ Since May 2016, CDTI control programmes are integrated in the WHO Expanded Special Project for Elimination of Neglected Tropical Diseases). ${ }^{30}$ CDTI overcomes the limited performance of weak health systems in rural areas by using an active strategic involvement of the community. ${ }^{31}$ To reach the entire population, ivermectin distribution is organised by trained volunteers in each village, resulting in a large geographical coverage. CDTI, in certain regions combined with the control of the blackfly by larviciding of breeding sites (ie, fast-flowing rivers), resulted in a measurable impact reflected by a remarkable transmission reduction in the past 20 years. ${ }^{32}$ However, success and effectiveness of these targeted interventions lack comprehensiveness. Certain onchocerciasis endemic regions remain undersupplied or unreachable for CDTI due to political instability, insecurity or armed conflicts. ${ }^{33}$ As a consequence in the war-affected regions of South Sudan and northern Uganda, onchocerciasis control measures were stopped or implementation started only recently. Moreover, misconceptions and the fear of adverse effects result in suboptimal therapeutic coverage and reduce the effectiveness of the control programme. ${ }^{34}$ Adding to the complexity of onchocerciasis control, in regions where Loa loa and onchocerciasis are coendemic, ivermectin may cause severe adverse effects (encephalopathy, coma or death) in infected individuals harbouring a high Loa loa microfilariae load. ${ }^{35}$ Thus, CDTI implementation 


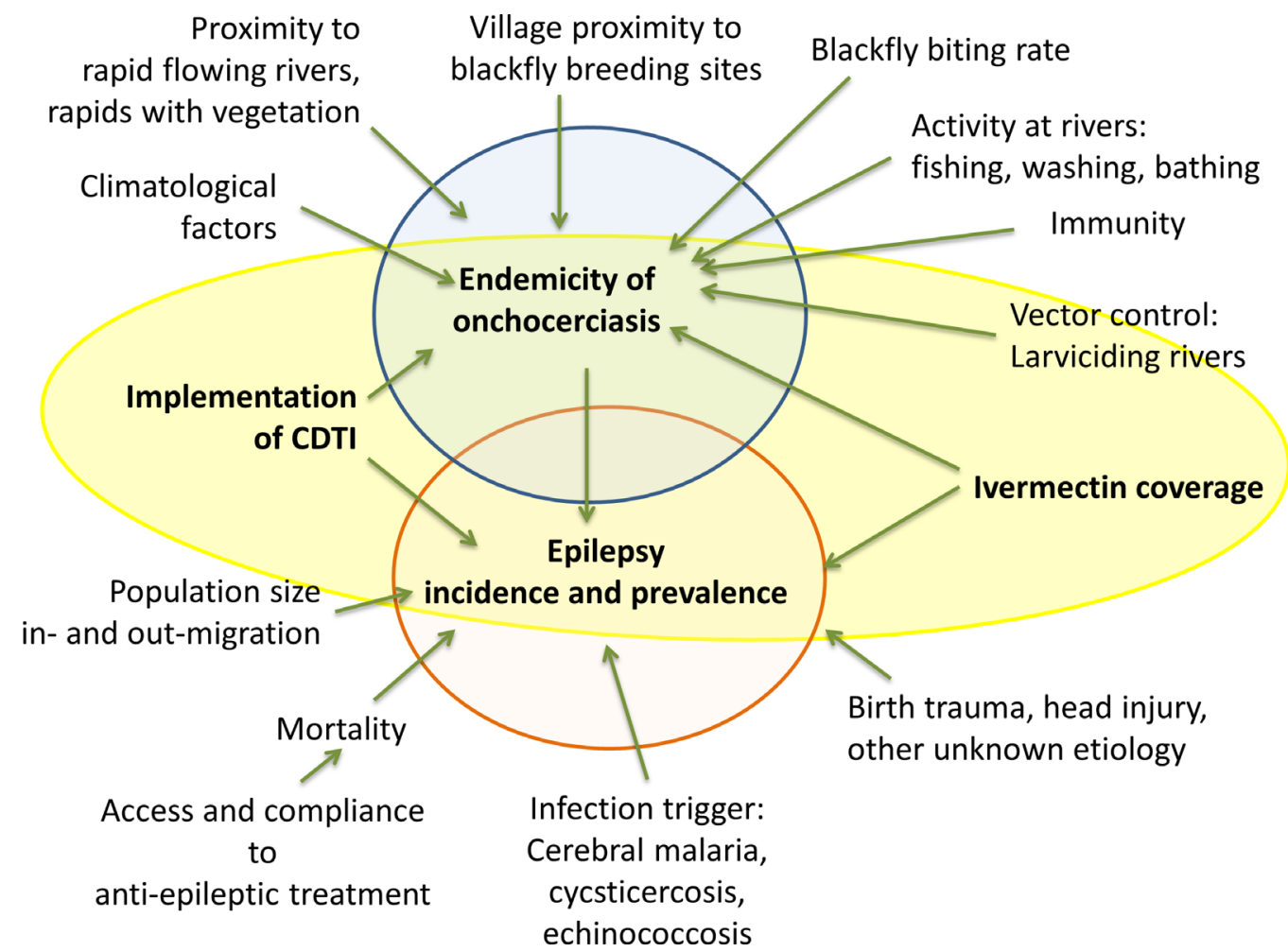

Figure 1 Onchocerciasis endemicity and prevalence and incidence of associated epilepsy are influenced by an extensive network of controllable and uncontrollable factors. CDTI, community-directed treatment with ivermectin.

in such regions requires additional precautions. ${ }^{37}$ This is the case in certain regions of Cameroon and the Democratic Republic of Congo (DRC) ${ }^{38}$ Compliance to CDTI programmes can also decrease over the years since less direct positive effects can be observed when onchocerciasis prevalence drops, and healthy feeling people may not appreciate the importance of continuing repeated treatment. $^{39}$

\section{The potential of CDTI to reduce the incidence of epilepsy in} onchocerciasis endemic regions

The endemicity of onchocerciasis and the prevalence and incidence of epilepsy are influenced by a multitude of factors (figure 1). These factors are of uncontrollable nature (climate, environment and ecology) and of controllable nature (prevention and intervention programmes, access to healthcare and treatment).

The two heavily affected regions of the last decades, northern Uganda and South Sudan, were-and South Sudan still is-affected by armed conflicts. In 2012, after the conflict ended in northern Uganda, the NS epidemic was investigated. Following on, epilepsy treatment was provided, biannual CDTI was implemented and larviciding of major rivers was carried out. ${ }^{40}$ Since 2013, the NS epidemic in northern Uganda has reportedly been halted. ${ }^{41}$ In contrast in South Sudan, where CDTI was stopped because of insecurity, new cases of NS continue to appear. ${ }^{18}$ Based on the observations from northern Uganda, it has been suggested that ivermectin may reduce the incidence of NS and other forms of epilepsy in onchocerciasis endemic areas. ${ }^{42}$ Considering that $30 \%$ of the globally 37 million individuals infected with onchocerciasis do not have access to effective treatment, and that $1 \%$ of those develop epilepsy (equivalent to the estimated excess prevalence of epilepsy over non-onchocerciasis areas), the number of excess cases of epilepsy attributed to onchocerciasis most likely exceeds $100000 .^{17}$

Recent observations in the DRC and northern Uganda suggest that optimal treatment coverage of ivermectin may stop the incidence of NS and other forms of OAE. ${ }^{42}$

\section{Aim}

The population-based study from 1989 by Rwiza $e t a t^{43}$ established the baseline prevalence of all forms of epilepsy in the Ulanga district, Tanzania. This population-based study aims to further evaluate the prevalence and incidence rates of new epilepsy cases, including a complete case ascertainment of all forms of epilepsy encountered and compare the data to the 1989 data (table 1).

\section{Objective}

The main objective of this study is to identify the potential impact of long-term onchocerciasis control using CDTI on the prevalence and incidence of epilepsy in selected villages in the Mahenge area of the Ulanga district in Tanzania.

\section{Specific objectives}

1. To determine the prevalence of all forms of epilepsy in selected villages of the Mahenge area and compare the related data from 2017 to the 1989 data; 
Table 1 The pre-CDTI and post-CDTI epilepsy study periods and estimated epilepsy prevalence and incidence in the Mahenge area, Ulanga district in Tanzania

\begin{tabular}{lll}
\hline \multicolumn{3}{l}{ Mahenge area, Ulanga district, Tanzania } \\
\hline $\begin{array}{l}\text { Year of epilepsy study } \\
\text { data collection }\end{array}$ & $1989^{43}$ & 2017 \\
$\begin{array}{l}\text { Status of CDTI at study } \\
\text { year }\end{array}$ & Pre-CDTI & $\begin{array}{l}\text { Post-CDTI } \\
\text { (ongoing since 1997) }\end{array}$ \\
$\begin{array}{lll}\text { Estimated epilepsy } \\
\text { prevalence }\end{array}$ & $20.2 / 1000$ & $14 / 1000^{*}$ \\
$\begin{array}{l}\text { Estimated epilepsy } \\
\text { incidence }\end{array}$ & $146 / 100000$ & $81.7 / 100$ 000† \\
\hline
\end{tabular}

${ }^{*}$ Median epilepsy prevalence.

†Incidence in low-income and middle-income countries. ${ }^{34}$

CDTI, community-directed treatment with ivermectin.

2. to determine the incidence rate of annual new onset cases of all forms of epilepsy in the Mahenge area and compare the related data from 2017 to the 1989 data;

3 . to assess the ivermectin coverage and the onchocerciasis prevalence with a rapid epidemiological mapping of onchocerciasis (REMO) assessment in selected villages of the Mahenge area;

4. to determine the level of onchocerciasis transmission by a serological survey using a rapid test among children in selected villages of the Mahenge area;

5. to investigate the potential difference in clinical appearance of epilepsy in patients with negative onchocerciasis serology to patients with positive onchocerciasis serology.

\section{METHODS AND ANALYSIS}

The study consists of two parts: (1) a study to determine the prevalence and incidence of epilepsy and (2) a study to determine ivermectin coverage, onchocerciasis prevalence and level of transmission. Data collection was carried out between January and September 2017. Analysis is planned to be finalised by end of 2017 to allow publishing of the results in 2018.

\section{Study site}

The study is taking place in the Mahenge area of the Ulanga distict, Morogoro region in southeastern Tanzania, a mountainous area with fast-flowing rivers.

\section{Epilepsy in the Mahenge area}

In the 1960s, Aall-Jilnek was the first to report a high burden of epilepsy in the Mahenge area and also described the first NS cases. ${ }^{10}$ In 1989, Rwiza et al carried out a population-based survey to determine prevalence and incidence of epilepsy in the Ulanga district and found an annual incidence of 73.3 new cases in 100000 . The prevalence was 10.2 cases in 1000 and varied between villages from 5.1 to 37.1 cases in 1000 . Those villages with the highest prevalence were located in the Mahenge highlands (table 2). ${ }^{43}$
Table 2 Population size and prevalence of epilepsy in some villages of the Ulanga district, Tanzania, showing the highest prevalence in 1989, as reported by Rwiza et al in 1992

\begin{tabular}{llll}
\hline $\begin{array}{l}\text { Name of } \\
\text { village }\end{array}$ & $\begin{array}{l}\text { Population } \\
\text { size }\end{array}$ & $\begin{array}{l}\text { Number of } \\
\text { epilepsy cases }\end{array}$ & $\begin{array}{l}\text { Prevalence per } \\
\mathbf{1 0 0 0} \text { people }\end{array}$ \\
\hline Sali* $^{*}$ & 1282 & 14 & 10.9 \\
Mdindo* $^{*}$ & 539 & 20 & 37.1 \\
Vigoi* $^{*}$ & 1822 & 23 & 12.6 \\
Lupiro & 1697 & 17 & 10 \\
Misegezi & 1667 & 18 & 10.8 \\
\hline Total & 7007 & 92 & 13.13 \\
\hline
\end{tabular}

*Located in Mahenge highlands.

\section{Onchocerciasis and CDTI in the Mahenge area}

The Morogoro region is among the five regions where onchocerciais is endemic in Tanzania. The region presents four foci: Uluguru mountain, Ulanga/Mahenge, Kilosa and the Nguru mountain foci. ${ }^{44}$ The Ulanga district, specifically the Mahenge area, was known for its high endemicity of onchocerciasis since the early last century. ${ }^{45}$ Mass drug administration using annual CDTI was introduced in the Mahenge area in $1997 .{ }^{46}$ Before CDTI was implemented, $58.6 \%$ of the 482 inhabitants of the Mahenge area tested were found to have a microfilariae-positive skin snip. ${ }^{47}$ Twelve years later (in 2009), the REMO in 10 villages in the Mahenge area revealed that the percentage of persons with a microfilariae-positive skin snip has dropped to $21.9 \%$, with a mean prevalence of $8.3 \%{ }^{32}$

\section{Study population}

The three villages, Mdindo, Vigoi and Misegezi, that had high epilepsy prevalence during the 1989 study will be selected for this study. ${ }^{43}$ The entire population of each of these villages will be included in the study.

\section{Epilepsy and NS prevalence/incidence study}

Study design

The study is designed as a population-based cross-sectional study, following a two-stage approach for epilepsy case identification at village level. The gold standard in neuroepidemiological surveys to identify epilepsy cases in LMICs, the door-to-door approach, will be applied. ${ }^{48}$ All inhabitants of the selected villages will be eligible for participation and will be included in the questionnaire screening survey. Due to well-described limitations of questionnaire studies on epilepsy (stigma leading to concealment of cases, recall bias, absence of clear terminology for epilepsy and seizures) and to increase sensitivity of case ascertainment, key informants who are likely to be aware of persons with epilepsy in the village will additionally be consulted. ${ }^{38}$ These may be health workers, traditional healers, teachers or community leaders. ${ }^{49}$

In a second stage, persons with suspected epilepsy identified during the household screening survey will be 
Table 3 List of the study villages in the Mahenge highlands, Tanzania, and their population size in 1989 and in 2016

\begin{tabular}{lll}
\hline Name of village & $\begin{array}{l}\text { Population size } \\
\text { (1989) }\end{array}$ & $\begin{array}{l}\text { Population size } \\
\text { (2016) }\end{array}$ \\
\hline Mdindo & 539 & 1536 \\
Vigoi & 1822 & 2572 \\
Misegezi & 1667 & $3658^{\star}$ \\
Total & 4028 & 7766 \\
\hline
\end{tabular}

*Population projection based on the growth rate of Vigoi village.

further invited for clinical examination by a neurologist. The examination will include neurological tests and a detailed interview for case verification. In verified epilepsy cases, the onset of the seizures will be determined and possible aetiological factors will be investigated, such as birth trauma, head injury, meningoencephalitis, cerebral malaria, neurocystocercosis and the history of onchocerciasis. For persons with suspected epilepsy, their serological status will be determined using onchocerciasis rapid diagnostic test (Ov16 RDT) (Standard Diagnostics, Gyeonggi-do, Republic of Korea).

\section{Sample size calculation}

According to Rwiza et al the average prevalence of epilepsy in five villages with high prevalence was $1.313 \%$ (table 2). If we assume a reduction in the prevalence by $33.3 \%$ to be able to compare the prevalence at a power of $80 \%$ and 95\% confidence level, the minimal sample size of 4746 individuals will be required. Assuming a participation of $80 \%$, a minimum source population of 5933 is necessary to obtain optimal sample size. Since 1989 , the population in the villages with high epilepsy prevalence has increased (table 3). For this survey, we will include only the population of the villages with the highest epilepsy prevalence in 1989. The total population of the selected villages of Mdindo, Vigoi and Misegezi in 2016 is about 7766.

\section{Data collection at community level}

The community survey will commence by a questionnaire interview with the village authorities on demographic topics and with village health workers to address general questions on the status of epilepsy and epilepsy treatment in the village. A complete door-to-door active screening for persons with suspected epilepsy at village level will be performed. The interview team will be trained on how to conduct the active search for epilepsy cases using a pretested, validated screening questionnaire targeting epilepsy by five specific questions (provided in the online supplementary material).$^{50}{ }^{51}$ For validation, the questionnaire will be translated to Kiswahili, pretested and retranslated to English. The locally used terms for the two respective conditions will be used (epilepsy: kifafa and NS: kusinzia kichwa). To ascertain completeness and to ensure the best collaboration with the village population, the interviewer team will be accompanied to all households by local village health workers. The geographical coordinates of the participating households will be collected for the mapping and geospatial analysis of cases (proximity to rivers and potential clustering).

\section{Case verification and validation}

All persons with suspected epilepsy identified during the door-to-door survey will be verified by a neurologist. The neurologist will perform a detailed anamnesis on all persons with suspected NS and epilepsy. In case of confirmation of the epilepsy, the neurologist will perform a medical examination and administer a detailed questionnaire on the type of epilepsy. Newly diagnosed epilepsy cases will be referred to an epilepsy treatment centre. In case the person is already followed in a treatment centre, permission will be asked to review the medical information available in the treatment centre to record epilepsy diagnosis and epilepsy treatment history.

\section{Definitions}

1. A case of epilepsy will be defined as a patient who had at least two times non-febrile seizures unrelated to any acute metabolic disorder or to withdrawal of alcohol or drugs, with a minimal time difference of 24 hours between the two events. This is in accordance to the current guidelines of the International League Against Epilepsy for an operational definition of epilepsy and to the definition used by Rwiza et al in their baseline study performed in 1989. ${ }^{43} 4952$

2. A case will be considered as active epilepsy if the patient is receiving epilepsy treatment or, if without antiepileptic treatment, the patient presented at least one seizure during the last 5 years.

3. A case of suspected NS will be defined as a person who presented with episodes of decreased consciousness during which the head dropped forward repeatedly.

4. New cases of epilepsy will be defined as cases that appeared within the last 12 months preceeding the study period.

\section{Onchocerciasis prevalence study \\ Study design}

This study includes two approaches. The aim of the first approach will be to determine the onchocerciasis prevalence in the selected villages after 20 years of CDTI by performing the WHO proposed REMO methodology. ${ }^{53}$ In brief, in each study village, 50 adults aged at least 20 years and resident in the community for at least 10 years will be invited to participate. They will be examined for the presence of onchocerciasis nodules (subcutaneous nodules or deep, painless, firm, mobile nodules located over bony prominences: pelvic girdle, costal grid, knees and skull). Although precontrol onchocerciasis prevalence data from the study villages is not available, a study from 1966 carried out in three villages in Mahenge found a skin snip positivity rate ranging from $43 \%$ to $65 \% .^{54}$

The second approach aims at determining the level of transmission of onchocerciasis in the selected villages. 
Table 4 Estimated number of children aged 7-10 years old in the study villages in the Ulanga district, Tanzania, in 2016

\begin{tabular}{llll}
\hline $\begin{array}{l}\text { Name of } \\
\text { village }\end{array}$ & $\begin{array}{l}\text { Population } \\
\text { size (2016) }\end{array}$ & $\begin{array}{l}\text { Estimated } \\
\text { population aged } \\
\text { 7-10years }\end{array}$ & $\begin{array}{l}\text { Estimation of } \\
\text { participating } \\
\text { children (80\%) }\end{array}$ \\
\hline Mdindo & 1536 & 178 & 143 \\
Vigoi & 2572 & 298 & 239 \\
Misegezi & $3658^{*}$ & $424^{*}$ & $340^{*}$ \\
Total & 7766 & 900 & 722 \\
\hline
\end{tabular}

${ }^{*}$ Population projection based on the growth rate of Vigoi village.

Therefore, serological testing for onchocerciasis will be done in all children aged 7-10 years, using the onchocerciasis specific Ov16 RDT (Standard Diagnostics). According to the National Census survey in 2012, the population of children aged 7-10 years represented $11.6 \%$ of the population in Ulanga district. Estimated population of children aged 7-10 years from the four selected villages is 900 , of which $722(\sim 80 \%)$ are anticipated to participate in the survey giving a power of $85 \%$ in detecting the prevalence between $0.8 \%$ and $2 \%$ at $5 \%$ significance level (table 4).

\section{Data collection procedures}

Data collection will be done using numeric tablets. All data collection forms will be developed in the opensource software 'Open Data Kit' (ODK, https://opendatakit.org/). Interviewers will be trained in how to perform tablet-based surveys. A technical data coordinator will be assigned to guarantee completeness and quality of data and to assure daily data transfer from each tablet to the central server for data security.

\section{Data management and analysis}

The prevalence of epilepsy and NS will be computed as a ratio of the number of epilepsy and NS cases per total number of people registered in the households visited, respectively. The incidence of new cases of epilepsy is defined as the number of persons who developed epileptic seizures within 2 years preceding the study, divided by twice the population size, assuming that the change in population within the 2 years has a minimal effect on the incidence. Results will be presented accompanied with $95 \% \mathrm{CI}$ and $\mathrm{P}<0.05$ level of significance. Prevalence and incidence will be compared between villages and with the 1989 data. Ivermectin treatment coverage and onchocerciasis prevalence will be calculated. Proportions will be compared using $\chi^{2}$ test, while means will be compared using t-tests. Demographic and clinical characteristics of persons with epilepsy having a positive Ov16 serology will be compared with epilepsy cases with negative Ov16 serology. Ivermectin treatment coverage, epilepsy prevalence and incidence and OV16 positivity rate among children aged 7-10 years will be compared among villages, weighted for the difference in population size between villages. ORs will be calculated for epilepsy cases with positive Ov16 results, and the association of Ov16 positivity and epilepsy will be analysed by age group.

\section{Ethics}

Before the activities start, the research team will hold meetings with community leaders and health workers of the selected villages. The procedure, purpose and specific aim of the study will be explained and discussed with regard to the potential risks and benefits for the community. Community leaders, village health workers and researchers will maintain the initially established communication for the entire duration of the study. The dissemination of results will be organised in a similar way as the initial meeting.

As approved by the relevant ethics committees, only participants who provide written informed consent will be enrolled in the study. Participant information sheets and consent forms will be available in English and Kiswahili. In case of illiteracy, information sheets and consent forms will be read to the participant in the presence of a witness. All participants will be permitted to withdraw from the study activities, without reason, at any time. All personal information, samples and test results will be encoded and treated confidentially. People identified with untreated epilepsy or with interrupted treatment will be referred to the treatment centre and will receive advice for care and support.

\section{Data storage and handling}

All data files will be centralised and stored in a secured central server. Name-linked information on participants and ID codes will remain confidential and will be used only to communicate clinical results to participants for their respective treatments.

\section{DISCUSSION}

The central research question of this study is to determine whether mass ivermectin administration using CDTI has the potential to prevent the onset of OAE. The expected results will contribute to a better understanding of the linkage between the onset of epilepsy and NS in particular, onchocerciasis and the impact of CDTI. In northern Uganda, an NS epidemic has been halted after introducing a programme combining CDTI and larviciding of the main rivers. This study will be the first to investigate systematically whether CDTI alone may reduce epilepsy in an onchocerciasis endemic region. To do so, pre-CDTI and post-CDTI epilepsy prevalence and incidence data will be compared. Concurrently, with the Ov16 serological survey among children aged 7-10 years, it will be possible to assess wether the transmission of onchocerciasis is ongoing in the Mahenge area after 20 years of CDTI. To date, the onchocerciasis control programme in the study area was monitored based on the annual data on total ivermectin distribution and the treatment coverage data provided by the community distributors. Also, ivermectin treatment coverage data will be assessed in the 
framework of this study by interviewing the population. Moreover, by performing an Ov16 seroprevalence study among children under 10 years of age, a real-time estimate of the level of ongoing transmission of onchocerciasis will be evaluated. The hypothesis is to find a low Ov16 seroprevalence in children and a decreased prevalence of epilepsy since 1989. In case a high Ov16 seroprevalence is found, this will suggest that the CDTI programme was performing suboptimal and/or that ivermectin resisitance may have developed. It might therefore be useful to combine CDTI with larviciding rivers to reduce blackfly abundance.

This study also has limitations. The methods used will not allow for measuring onchocerciasis infection intensity, one of the main factors influencing the development of OAE. There is a possibility that it may not be possible to show an impact of CDTI because, in case of highlevel exposure to infectious blackflies, the administration of ivermectin only once a year may not be sufficient to considerably decrease onchocerciasis transmission. Moreover, in pre-CDTI and post-CDTI comparison, it is possible that observed differences in epilepsy prevalence and incidence are not related to the intervention (CDTI) but to some of the other factors (eg, those mentioned in figure 1) that might have changed over time. However, a site visit to the Mahenge study site revealed that the village population had increased by a factor of 3 , but there was no important inmigration or outmigration or any other major change in lifestyle of the population or another major environmental change. In all the villages included in the study, such potential changes will be carefully assessed to control for potential confounding factors. Families who migrated into the study area after the implementation of CDTI will not be included in the analyses.

\section{Outlook}

This study aims to unravel the potential impact of CDTI on reducing the incidence of epilepsy in onchocerciasis endemic areas. Study results may provide evidence that strengthening CDTI programmes could prevent the onset of OAE. The results and lessons learnt from this study will be disseminated by publications in open-access journals, as well as presentations at scientific conferences and shared with all interested health authorities in Tanzania and beyond.

Acknowledgements We are grateful to Dr Alfred Kilimba and Dr Yohanna Mahenda, Epilepsy Clinic Mahenge, Tanzania, and the health authorities in Vigoi, Mbindo and Misegezi for the fruitful discussions during our visit and for their input that provided background information and practical considerations for the development of the current study protocol.

Contributors All listed authors contributed to the development of the study design, essential study documents and study tools. According to their different areas of expertise, the authors critically revised specific parts of this manuscript: HG wrote the manuscript; HG, BM, PS and RC developed the study protocol; WilliamsM, MM, WilliamM developed and approved the neurological study protocol and the survey tools; HG, BM and AK developed the methodology and tools for the digital data collection; $\mathrm{HG}, \mathrm{BM}$ and $\mathrm{RC}$ visited the study sites.
Funding This research is embedded in a five-country research project on epilepsy, nodding syndrome and onchocerciasis entitled 'NSETHIO', and receives funding from the European Research Council, Advanced Grant (ERC-2014-ADG), grant no. 671055.

Competing interests None declared.

Patient consent Not required.

Ethics approval The protocol has received ethical approval from the ethics committee of the University of Antwerp, Antwerp, Belgium (29 August 2016) and the National Institute of Medical Research (NIMR) ethical committee Dar es Salaam, Tanzania (24 August 2016).

Provenance and peer review Not commissioned; externally peer reviewed.

Open Access This is an Open Access article distributed in accordance with the Creative Commons Attribution Non Commercial (CC BY-NC 4.0) license, which permits others to distribute, remix, adapt, build upon this work non-commercially, and license their derivative works on different terms, provided the original work is properly cited and the use is non-commercial. See: http://creativecommons.org/ licenses/by-nc/4.0/

(c) Article author(s) (or their employer(s) unless otherwise stated in the text of the article) 2018. All rights reserved. No commercial use is permitted unless otherwise expressly granted.

\section{REFERENCES}

1. WHO. Investing to overcome the global impact of neglected tropical diseases. Third WHO report on neglected tropical diseases. Geneva: World Health Organization, 2015.

2. Paul A, Adeloye D, George-Carey R, et al. An estimate of the prevalence of epilepsy in Sub-Saharan Africa: A systematic analysis. $J$ Glob Health 2012;2:020405.

3. Ngugi AK, Kariuki SM, Bottomley C, et al. Incidence of epilepsy: a systematic review and meta-analysis. Neurology 2011;77:1005-12.

4. Ngugi AK, Bottomley C, Kleinschmidt I, et al. Estimation of the burden of active and life-time epilepsy: a meta-analytic approach. Epilepsia 2010;51:883-90.

5. Newton CR, Garcia HH. Epilepsy in poor regions of the world. Lancet 2012;380:1193-201.

6. Ngugi AK, Bottomley C, Kleinschmidt I, et al. Prevalence of active convulsive epilepsy in sub-Saharan Africa and associated risk factors: cross-sectional and case-control studies. Lancet Neurol 2013;12:253-63.

7. Pion SD, Kaiser C, Boutros-Toni F, et al. Epilepsy in onchocerciasis endemic areas: systematic review and meta-analysis of populationbased surveys. PLoS Negl Trop Dis 2009;3:e461.

8. Kamuyu G, Bottomley C, Mageto J, et al. Exposure to multiple parasites is associated with the prevalence of active convulsive epilepsy in sub-Saharan Africa. PLoS Negl Trop Dis 2014;8:e2908.

9. Moshé SL, Perucca E, Ryvlin P, et al. Epilepsy: new advances. Lancet 2015;385:884-98.

10. Aall-Jilek LM. Epilepsy in the Wapogoro tribe in Tanganyika. Acta Psychiatr Scand 1965;41:57-86.

11. Jilek-Aall L. Epilepsy and Onchocerciasis: Pioneering research of Mexican physicians vindicated. Investigación en Salud 2004; $\mathrm{VI}(1: 22-7$.

12. Dowell SF, Sejvar JJ, Riek L, et al. Nodding syndrome. Emerg Infect Dis 2013;19:1374-1373.

13. Kaiser C, Rubaale T, Tukesiga E, et al. Nodding syndrome, western Uganda, 1994. Am J Trop Med Hyg 2015;93:198-202.

15. lyengar PJ, Wamala J, Ratto J, et al. Prevalence of nodding syndrome--Uganda, 2012-2013. MMWR Morb Mortal Wkly Rep 2014;63:603-6.

15. Winkler AS, Friedrich K, König R, et al. The head nodding syndrome--clinical classification and possible causes. Epilepsia 2008;49:2008-15.

16. Tumwine JK, Vandemaele K, Chungong S, et al. Clinical and epidemiologic characteristics of nodding syndrome in Mundri County, southern Sudan. Afr Health Sci 2012;12:242-8.

17. Foltz JL, Makumbi I, Sejvar JJ, et al. An Epidemiologic Investigation of Potential Risk Factors for Nodding Syndrome in Kitgum District, Uganda. PLoS One 2013;8:e66419.

18. Colebunders R, Hendy A, Mokili JL, et al. Nodding syndrome and epilepsy in onchocerciasis endemic regions: comparing preliminary observations from South Sudan and the Democratic Republic of the Congo with data from Uganda. BMC Res Notes 2016;9:182. 
19. Rafael F, Houinato D, Nubukpo P, et al. Sociocultural and psychological features of perceived stigma reported by people with epilepsy in Benin. Epilepsia 2010;51:1061-8.

20. Nakigudde J, Mutamba BB, Bazeyo W, et al. An exploration of caregiver burden for children with nodding syndrome (lucluc) in Northern Uganda. BMC Psychiatry 2016;16:255.

21. Ovuga E, Kipp W, Mungherera M, et al. Epilepsy and retarded growth in a hyperendemic focus of onchocerciasis in rural western Uganda. East Afr Med J 1992;69:554-6.

22. Kipp W, Kasoro S, Burnham G. Onchocerciasis and epilepsy in Uganda. Lancet 1994;343:183-4.

23. Boussinesq M, Pion SD, Demanga N, et al. Relationship between onchocerciasis and epilepsy: a matched case-control study in the Mbam Valley, Republic of Cameroon. Trans R Soc Trop Med Hyg 2002;96:537-41.

24. Kaiser C, Pion SD, Boussinesq M. Case-control studies on the relationship between onchocerciasis and epilepsy: systematic review and meta-analysis. PLoS Negl Trop Dis 2013;7:e2147.

25. Johnson TP, Tyagi R, Lee PR, et al. Nodding syndrome may be an autoimmune reaction to the parasitic wormOnchocerca volvulus. Sci Transl Med 2017;9:eaaf6953.

26. González P, González FA, Ueno K. Ivermectin in human medicine, an overview of the current status of its clinical applications. Curr Pharm Biotechnol 2012;13:1103-9.

27. Crump A, Ōmura S. Ivermectin, 'wonder drug' from Japan: the human use perspective. Proc Jpn Acad Ser B Phys Biol Sci 2011;87:13-28

28. Panic G, Duthaler U, Speich B, et al. Repurposing drugs for the treatment and control of helminth infections. Int $J$ Parasitol Drugs Drug Resist 2014;4:185-200.

29. Zouré HG, Noma M, Tekle AH, et al. The geographic distribution of onchocerciasis in the 20 participating countries of the African Programme for Onchocerciasis Control: (2) pre-control endemicity levels and estimated number infected. Parasit Vectors 2014:7:326.

30. Hopkins AD. Neglected tropical diseases in Africa: a new paradigm. Int Health 2016;8 Suppl 1(Suppl 1):i28-i33.

31. Amazigo UV, Leak SG, Zoure HG, et al. Community-driven interventions can revolutionise control of neglected tropical diseases. Trends Parasitol 2012;28:231-8.

32. Tekle AH, Zouré HG, Noma M, et al. Progress towards onchocerciasis elimination in the participating countries of the African Programme for Onchocerciasis Control: epidemiological evaluation results. Infect Dis Poverty 2016;5:66.

33. Lee BX, Leckman JF, Khoshnood K. Violence, health, and SouthNorth collaboration: Interdisciplinary research in light of the 2030 Agenda. Soc Sci Med 2015;146:236-42.

34. Senyonjo L, Oye J, Bakajika D, et al. Factors Associated with Ivermectin Non-Compliance and Its Potential Role in Sustaining Onchocerca volvulus Transmission in the West Region of Cameroon. PLoS Negl Trop Dis 2016;10:e0004905.

35. Zouré HG, Wanji S, Noma M, et al. The geographic distribution of Loa loa in Africa: results of large-scale implementation of the Rapid Assessment Procedure for Loiasis (RAPLOA). PLoS Negl Trop Dis 2011;5:e1210.
36. Boussinesq M, Gardon J, Gardon-Wendel N, et al. Clinical picture, epidemiology and outcome of Loa-associated serious adverse events related to mass ivermectin treatment of onchocerciasis in Cameroon. Filaria J 2003;2 Suppl 1(Suppl 1):S4.

37. WHO. Preventive chemotherapy in human helminthiasis. Coordinated use of anthelminthic drugs in control interventions: a manual for health professionals and programme managers. Geneva, Switzerland: World Health Organization, 2006.

38. Makenga Bof JC, Maketa V, Bakajika DK, et al. Onchocerciasis control in the Democratic Republic of Congo (DRC): challenges in a post-war environment. Trop Med Int Health 2015;20:48-62.

39. Utzinger J, Becker SL, Knopp S, et al. Neglected tropical diseases: diagnosis, clinical management, treatment and control. Swiss Med Wkly 2012;142:W13727.

40. Idro R, Musubire KA, Byamah Mutamba B, et al. Proposed guidelines for the management of nodding syndrome. Afr Health Sci 2013;13:219-32.

41. Wamala JF, Malimbo M, Tepage F, et al. Nodding Syndrome May Be Only the Ears of the Hippo. PLoS Negl Trop Dis 2015;9:e0003880.

42. Colebunders R, Irani J, Post R. Nodding syndrome-we can now prevent it. Int J Infect Dis 2016;44:61-3.

43. Rwiza HT, Kilonzo GP, Haule J, et al. Prevalence and incidence of epilepsy in Ulanga, a rural Tanzanian district: a community-based study. Epilepsia 1992;33:1051-6.

44. Wegesa P. The present status of onchocerciasis in Tanzania. A review of the distribution and prevalence of the disease. Trop Geogr Med 1970;22:345-51.

45. Geigy R, Colas J, Fernex M. Endemic onchocherciasis in the Ulanga area, Tanzania. Acta tropica 1965;22:70-3.

46. König R, Nassri A, Meindl M, et al. The role of Onchocerca volvulus in the development of epilepsy in a rural area of Tanzania. Parasitology 2010;137:1559-68.

47. Mwaiko GL, Mtoi RS, Mkufya AR. Onchocerciasis prevalence in Tanzania. Cent Afr J Med 1990;36:94-6.

48. Bharucha N, Odermatt P, Preux PM. Methodological difficulties in the conduct of neuroepidemiological studies in low- and middle-income countries. Neuroepidemiology 2014;42:7-15.

49. Thurman DJ, Beghi E, Begley CE, et al. Standards for epidemiologic studies and surveillance of epilepsy. Epilepsia 2011;52 Suppl 7(Supp 7):2-26.

50. Diagana M, Preux PM, Tuillas M, et al. [Dépistage de l'épilepsie en zones tropicales: validation d'un questionnaire en Mauritanie]. Bull Soc Pathol Exot 2006;99:103-7.

51. Preux PM. Questionnaire d'investigation de l'epilepsie dans les pays tropicaux. Bulletin de la Societe de pathologie exotique (1990) 2000;93:276-8.

52. Hauser WA, Annegers JF, Kurland LT. Prevalence of epilepsy in Rochester, Minnesota: 1940-1980. Epilepsia 1991;32:429-45.

53. Ngoumou P, Walsh JF, Mace JM. A rapid mapping technique for the prevalence and distribution of onchocerciasis: a Cameroon case study. Ann Trop Med Parasitol 1994;88:463-74.

54. Häusermann W. On the biology of Simulium damnosum Theoblad, 1903 , the main vector of onchocerciasis in the Mahenge mountains, Ulanga, Tanzania. Acta Trop 1969;26:29-69. 